LBL-37346

SC-MAG-503

\title{
A 6.3 T Bend Magnet for the Advanced Light Source*
}

\author{
Clyde E. Taylor and Shlomo Caspi \\ Lawrence Berkeley Laboratory \\ University of California \\ Berkeley, California
}

June 7, 1995

* This work was supported by the Director, Office of Energy Research, U.S. Department of Energy, under Contract No. DE-AC03-76SF00098 through Laboratory-Directed Research and Development funds. 


\title{
A 6.3 T Bend Magnet for the Advanced Light Source*
}

\author{
Clyde E. Taylor and Shlomo Caspi \\ Lawrence Berkeley Laboratory, Berkeley, California, 94720 USA
}

\begin{abstract}
The Advanced Light Source (ALS) is a 1.5 to 1.9 GeV high-brightness electron storage ring operating at LBL that provides synchrotron radiation for a large variety of users. It is proposed to replace three of the thirty six 1.5T, one meter long bend magnets with very short high-field superconducting (SC) dipoles. These magnets would provide bend-magnet synchrotron radiation to six beamlines with a critical energy of at least $6 \mathrm{keV}$ that is much better suited for protein crystallography and other small-sample $x$-ray diffraction and adsorption studies, than is currently available at the ALS. The magnet design is described, including coil, yoke, magnetic field analysis, and cyrostat. A prototype magnet is under construction at LBL.
\end{abstract}

\section{INTRODUCTION}

The superconducting magnets must be installed at three symmetrical locations without removing the existing ALS vacuum beamline; this leads to a $C$-shaped iron yoke.

The 10 degree bend requires an integrated field of 870 $\mathrm{T}-\mathrm{mm}$ (at $1.5 \mathrm{GeV}$ ); also the field at the 2.5 degree bend point must be at least $4 \mathrm{~T}$ (at $1.5 \mathrm{GeV}$ ) since radiation from this point is to be admitted to the experimental beamlines. This forces the magnet to be very short, (about $220 \mathrm{~mm}$ ) and, therefore, end effects are very important and will be influenced by the gap. To minimize the gap, material will be removed from the aluminum beam line walls to bring the pole faces as close together as possible; for the minimum warm gap of $53 \mathrm{~mm}$, the cold gap is $94 \mathrm{~mm}$ allowing a $20.5 \mathrm{~mm}$ space for vacuum wall, heat shield, and space for multi-layer insulation.

The ALS is injected at $1.5 \mathrm{GeV}$; for $1.9 \mathrm{GeV}$ operation the magnets must be ramped in a period of about 5 minutes.

\section{MAGNET}

\section{A. Coil Design}

The C-shaped cold iron yoke has a pair of "racetrack" coils at the pole. Central field on axis is $4.7 \mathrm{~T}$ at $1.5 \mathrm{GeV}$ and is ramped to $6.3 \mathrm{~T}$ for $1.9 \mathrm{GeV}$. The coil is graded into high field and low field sections for efficient use of superconductor. (The conductor parameters are similar to those for the main dipoles of the recently discontinued SSC magnets.) The strands are insulated with $13 \mu \mathrm{m}$ kapton, fiberglass is placed between each layer, and the coil is impregnated with epoxy to

- Supported by the Director, Office of Energy Research, of the U. S. Department of Energy, under Contract No. DE-AC03-76SF00098 through Laboratory-Directed Research and Development funds. Manuscript recieved June 13, 1995.
TABLE I Coil Parameters

$\begin{array}{lll} & \begin{array}{l}\text { Inner } \\ \text { layer }\end{array} & \begin{array}{l}\text { Outer } \\ \text { layer }\end{array} \\ \text { strand id. mm } & 0.81 & 0.65 \\ \text { Cu/SC ratio } & 1.3: 1 & 1.8: 1 \\ \text { strand insul. thickness mm } & 0.03 & 0.03 \\ \text { layer-layer insulation mm } & 0.10 & 0.20 \\ \text { coil packing fraction } & 0.69 & 0.62 \\ \text { width mm } & 65 & 65 \\ \text { number of layers } & 24 & 52 \\ \text { radial thickness mm } & 20.57 & 39.76 \\ \text { total tums } & 1812 & 4810 \\ \text { operating current A (at } 1.9 \mathrm{GeV} \text { ) } & 114 & 114 \\ \text { B max. at cond. T (at } 1.9 \mathrm{GeV} \text { ) } & 8.12 & 6.15 \\ \mathrm{~J} \text { overall A/sq. mm (at } 1.9 \mathrm{GeV} \text { ) } & 154 & 212 \\ \text { Jc at } 4.5 \mathrm{~K} \text { A/sq. mm (at } 1.9 \mathrm{GeV} \text { ) } & 818 & 1874 \\ \text { J overall / Jc (at } 1.9 \mathrm{GeV}) & 1.60 & 1.95\end{array}$

make a rigid structure. Table 1 shows coil parameters. The very constricted space in the gap makes it difficult to encase the coil in a container of liquid helium. Therefore, the coil is conduction cooled; copper plates, attached to the coil exterior, are cooled by liquid helium. Because of the indirect cooling method and the non-circular shape with large Lorentz forces, a low overall current density and a large operating "margin" were chosen. Operating current is low, $114 \mathrm{~A}$, to minimize lead heat leak; thus, the magnet will have a relatively high inductance, $27 \mathrm{H}$, and must be subdivided with shunts and diodes for quench protection.

There will be a persistent switch to allow current to be switched off during beam storage periods of several hours. However, the leads remain in place to allow convenient ramping between $1.5 \mathrm{GeV}$ and $1.9 \mathrm{GeV}$.

\section{B. Forces and Structure}

Fig. 1 is an equilibrium diagram of one quarter of a coil considered as a rigid body showing the total tensile forces in the windings required if the coil were self-supporting. Since the non-circular coil cannot be entirely self supporting without sustaining undesirable flexural stresses, it is contained by a $20 \mathrm{~mm}$ thick, $190 \mathrm{~mm}$ OD. aluminum alloy hoop which encircles the coil. Spacers carry the radial compressive forces between the non-circular coil outer layer and the hoop id. This arrangement relieves the coil of flexural stresses. The 


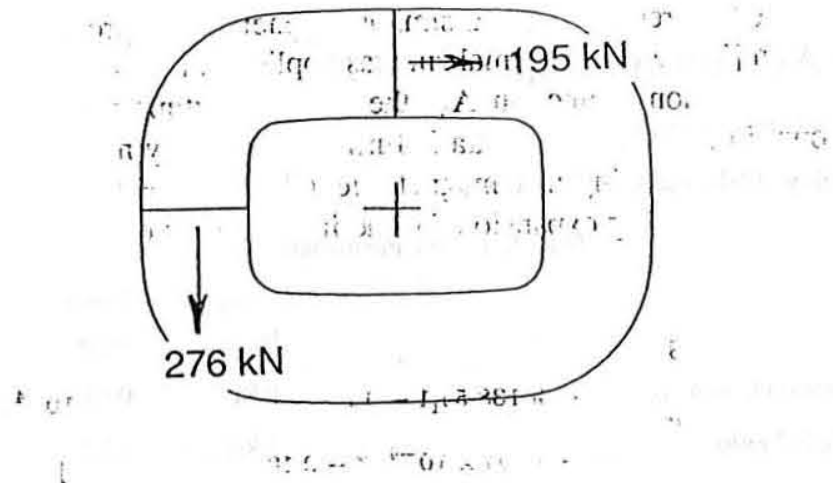

Fig. 1. Tensile forces in the windings for a self-supporting coil.

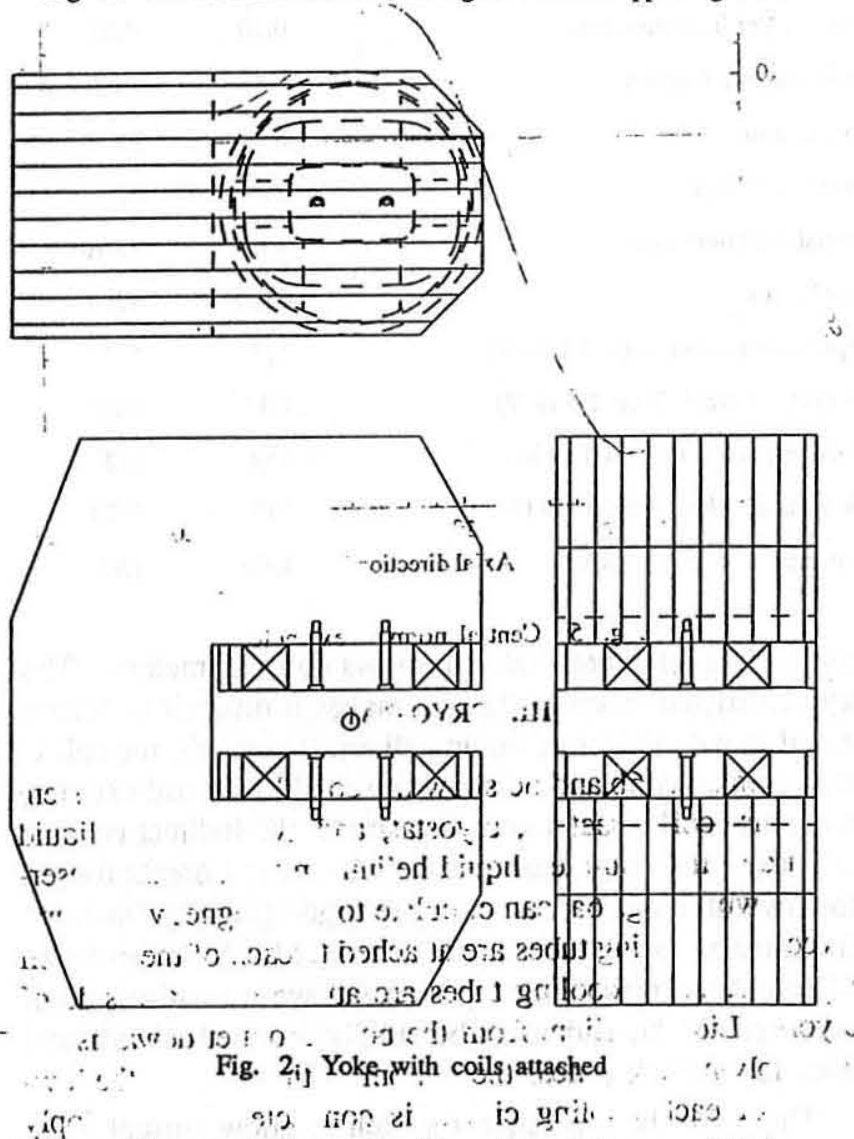

coil assembly consisting of coil, spacers; hoop, arilifcopper heat drain, is attached directly to the iron yoke. There is an vertical attractive force of $146 \mathrm{kNlbetween}$ each coil and the

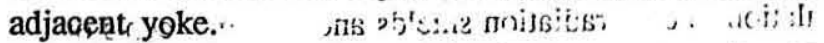
$-\mathrm{T} \quad \mathrm{O}$
C. Yoke 303
303 0, ?

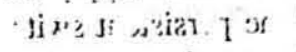

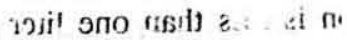

The' $\mathrm{C}$-shaped iron yoke weighs approximately $1500 \mathrm{~kg}$ and is laminated to suppress eddy-cturrënt heating during ramping from 1.5 to $1.9 \mathrm{Gev}$ ! 'Near the pole the the iron is completely.saturated and thietefore there is a significant fringing field; however, the ron criyostat wall confines the fringe field to the vicinity of the gap? The poleffiton is included as part of the coil assembly: Fig. $\mathcal{Z}$ showws the youke with coils

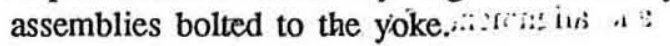
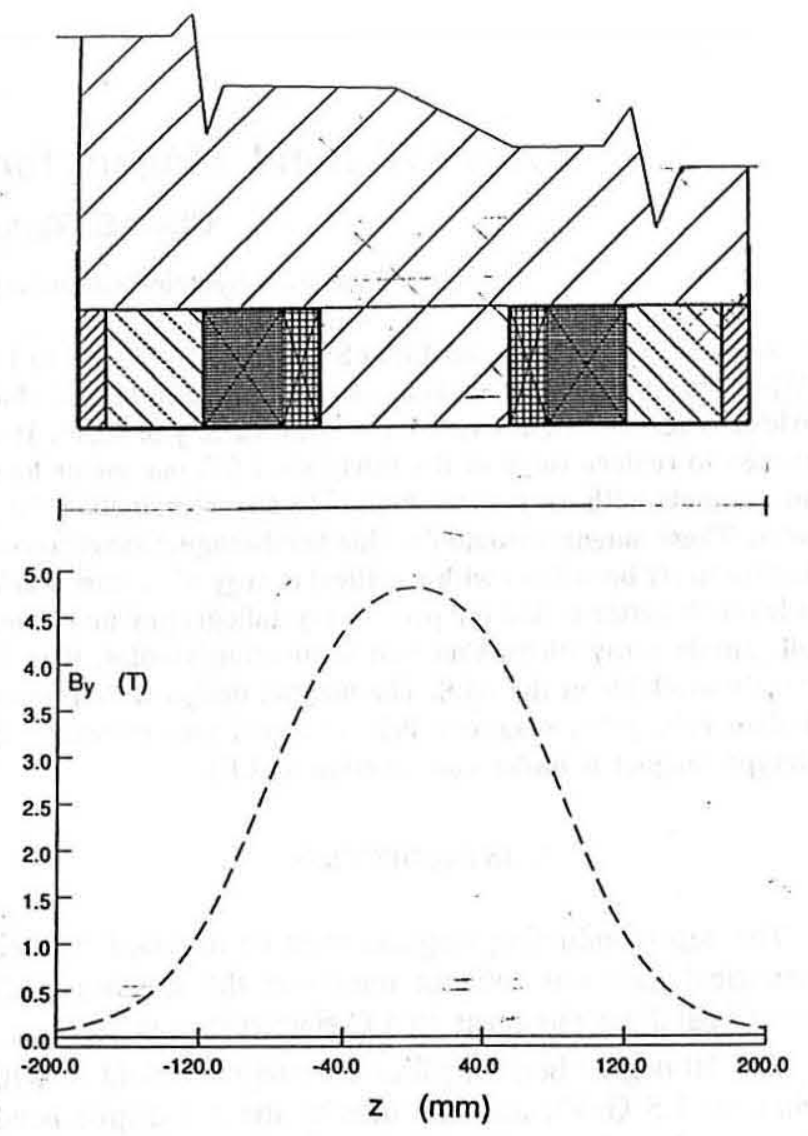

Fig. 3. Vertical field along the axis of the beam compared with a vertical cross-section of the coil and pole. '

i.).

\section{Magnetic Design}

Fig. 3 shows the vertical field along the beam for 1.5 $\mathrm{GeV}$. As seen in Fig. 3 there is no uniform field region because the magnet is very short. (A longer magnet would produce excess bending of the electron beam.) Nevertheless, the integrated magnetic field must be sufficiently uniform so that the ALS dynamic beam aperture and beam lifetime are not degraded. This can be done by extending the coils and yoke normal to the beam to make the field more uniform in the tran'sverse direction. However, since the yoke weight, stored,energy, andiforces increase with extension of the "long" side of the coil, it must be kept as small as possible. After studying the field uniformity of several options [1], the iron pole width of $180 \mathrm{~mm}$ was selected. Fig. 4 shows the vertical field at $1.5 \mathrm{GeV}$ along a line transvertse to the beam at the magnet center.

The multipole expansion in a short magnet such as Superbend may require a special 3 dimensional treatment like the one devised in Reference [2]. However, simulation of ALS beam optics permits the use of the thin lens approximation, so we may calculate multipole coefficients based on their integral value with respect to the axial coordinate $Z$ ( in an analogous manner, not applied here, one may integrate the current density first an then proceed with a 2 D harmonic analysis). As shown in the references cited above we may express the dipole field at any point on the $\mathrm{y}=0$ plane, as an expansion 

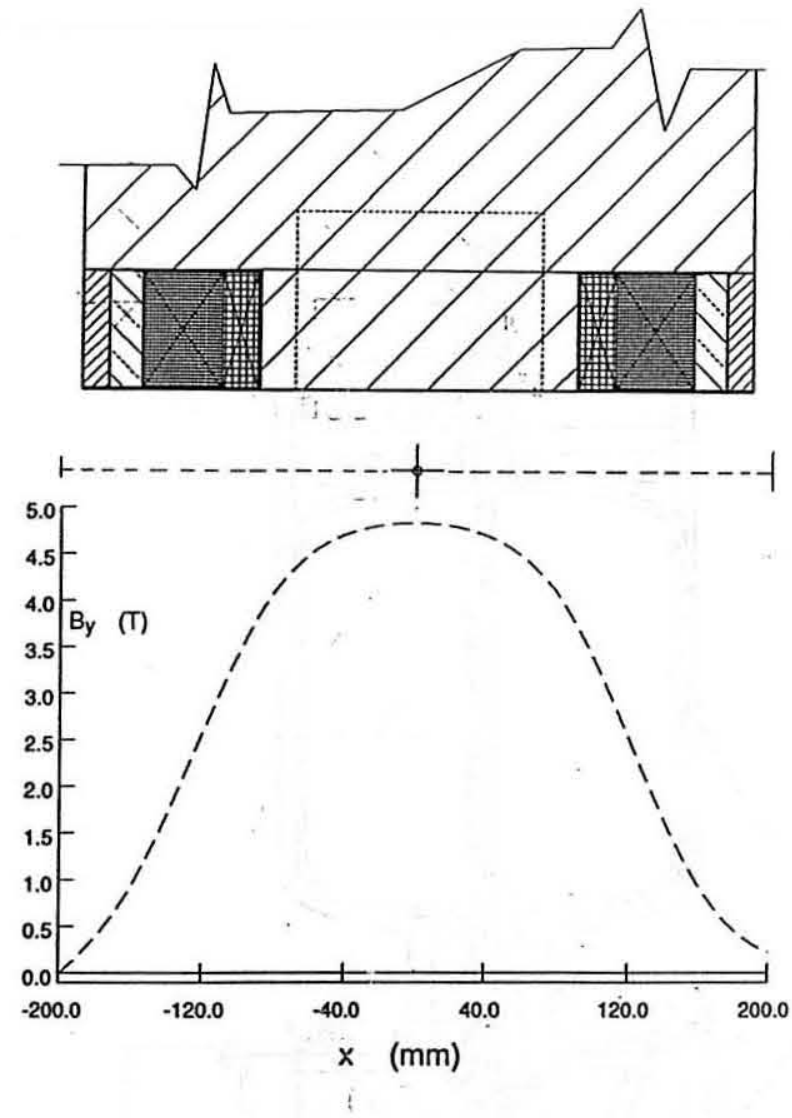

Fig. 4. Vertical field along a line transverse to the beam at the magnet center compared with a transverse cross-section of the coil and pole.

in $\mathrm{X}$ of harmonic coefficients that are a function of $\mathrm{Z}$,

$$
B_{y}(x, 0, z)=\sum_{n=1} \sum_{k=0}-(-1)^{k+1 ! 1} \frac{n ! n}{2^{2 k} k !(n+k) !} A_{n}^{(2 k)}(z) x^{2 k+n-1}
$$

where $\mathrm{k}$ denotes the order of differentiation of the functions $A_{n}$ with respect to $Z$. In a thin lens approximation we both integrate the above expression with respect to $\mathrm{Z}$ and normalize it with respect to the central field at $Z=0$, hence reducing the dipole field to a function of $\mathrm{X}$ only (we note that such an integration reduces to 0 all pseudo harmonics for which $: \mathrm{k}>0$ $\left.\int_{-\infty}^{+\infty} A_{n}^{(2 k)}(z) d z=0\right)$. With the definition ofuthe margnetic length $\mathrm{L}_{\mathrm{m}}$

$$
L_{m}=\frac{\int_{-\infty}^{\infty} B_{y}(0,0, z) d z}{B_{y}(0,0,0)}=\frac{\int_{-\infty}^{\infty} A_{1}(z) d z \text {. }}{A_{1}(0)}
$$

the above expression may be conveniently reduced to

$\overline{B_{y}}(x)=\frac{\int_{-\infty}^{\infty} B_{y} d z}{L_{m}}=-A_{1}(0)\left[1+2 \frac{\int A_{2}(z) d z}{B_{y}(0) L_{m}} x+3 \frac{\int A_{3}(z) d z}{B_{y}(0) L_{m}} x^{2}+\cdots\right]$

We have used the program TOSCA to evaluate the magnetic field on cylindrical grid ( $\mathrm{r}=20 \mathrm{~mm}, \mathrm{z}$ between $-50, \mathrm{~mm}$ and $300 \mathrm{~mm}$ with $1 \mathrm{~mm}$ increments and $\theta$ between $5^{\circ}$ and $175^{\circ}$ with $10^{\circ}$ increments). In a similar manner to the procedure cited in [3] the above formalism was applied in the evaluation of the harmonic function $A_{n}$ (the normal sextupole, $A_{3}$, is shown in , Fig. 5). The data is further reduced by numerical integration resulting in a magnetic length of $L_{m}=18.13 \mathrm{~cm}$ and the following expansion for the integral dipole field (cgs units):

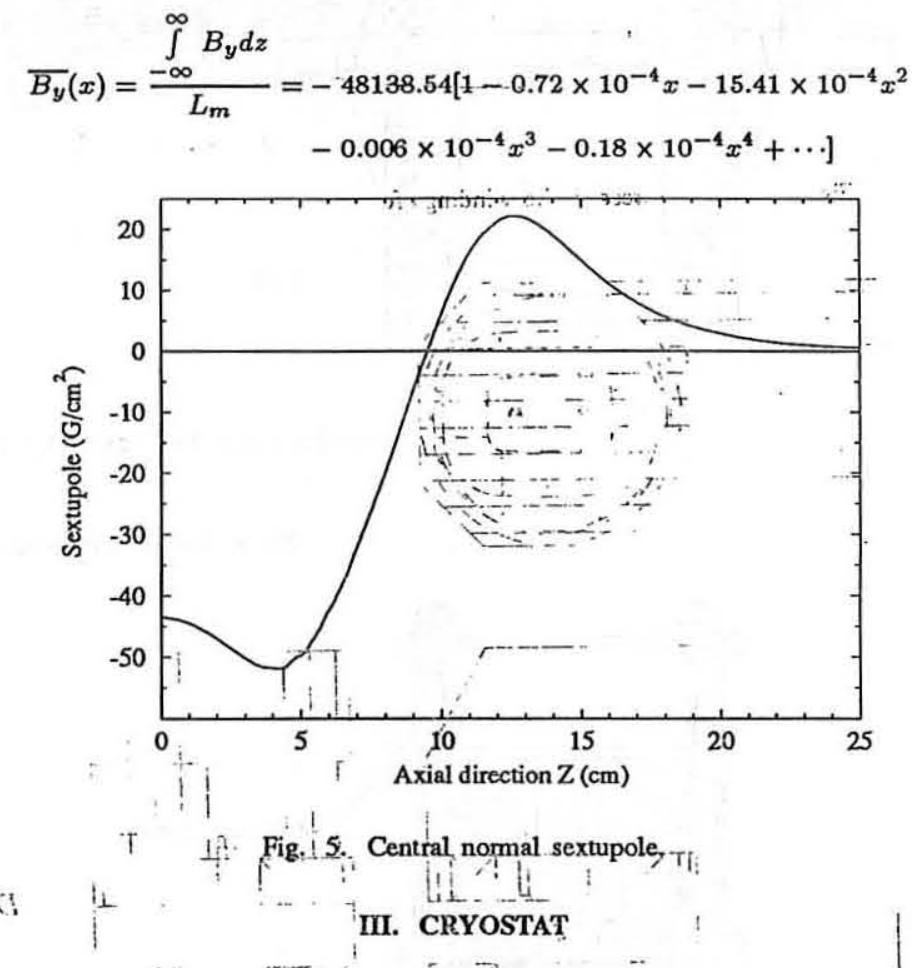

Figures $6 \mathrm{a}, 66$ and $6 \mathrm{c}$ show the complète magnet system with coils, coil strueture, cryostat, and bottom-outlet liquid, helium 'dewar. Saturated liquid helium in the 300 liter reservoir, shown in Fig $6 \mathrm{a}$, can circulate to the magnet via natural convection. Cooling tubes are attached to each of the two coil assemblies. Also, cooling tubes $\backslash$ are attached to each side of the yoke. Liquid helium from the bottom-outlet dewar is fed to a supply manifold neari the bottom of the magnet; the lowest end of each cooling circuit is connected to the supply manifold and the uppexend is connected to a retum manifold from which the vapor ean return to the reservoir. Similarly, liquid nitrogen is fed!from the initrogen reservoir via natural circulation to cool radiation shields and heat intereepts on the support straps. The supply dewar also contains the current leads and the persistent switch. Projected liquid helium consumption is less than one liter per hour. The yoke with coils attached weighs, about $1600 \mathrm{~kg}$ and is suspended by four

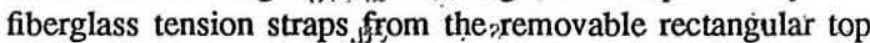
cover of the cryostat; four additional smaller downward straps " "i are added for preloading and stability against seismic loads: $:$ i The cryostat vacuum tank is $a_{5}$ steel box with non-magnetic $i \mathrm{i}$ : "slot" in the cryostat wall to accommodate the beam line; the : $\mathrm{r}$ ". part of the vacuum box containing the "slot" is removable for : ease of installation and alignment of the cold mass 


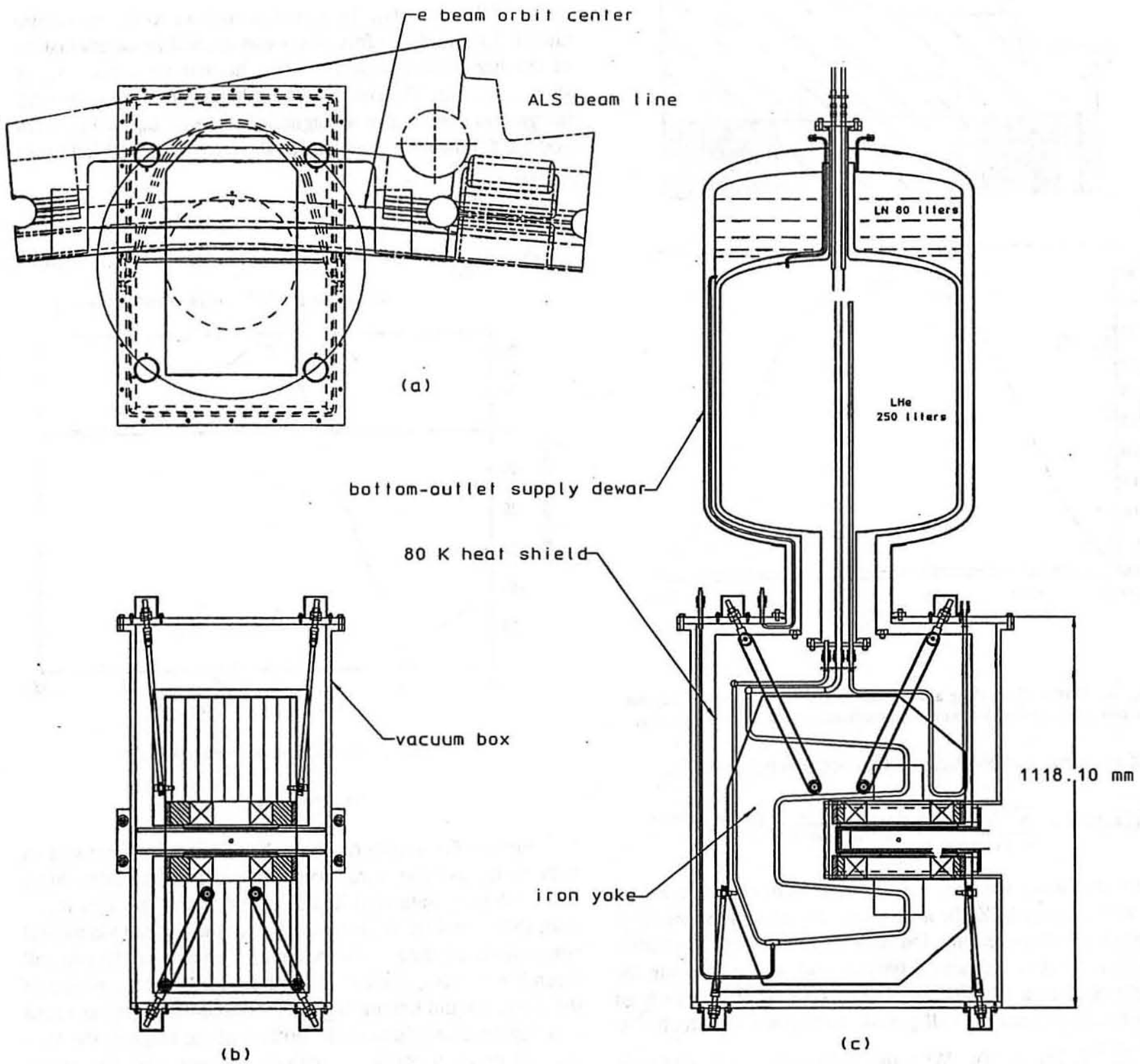

Fig. 6. (a) Top view showing installation in the ALS beamline., (b) Side view and (c) end view showing yoke, support straps, cryostat, and bottom outlet dewar

\section{STATUS}

Prototype coils are being fabricated and will be tested in about 3 months in a special test yoke. The complete prototype magnet is scheduled to be tested in about one year.

\section{ACKNOWLEDGMENT}

The authors acknowledge the significant contributions of Julian Cortella, Larry Morrison, and Richard Schafer, to the design of this magnet system.

\section{REFERENCES}

[1] C. Taylor and S. Caspi, "Field quality of 4 superbend magnet configurations," SC-MAG-493, February 1995.

[2] S. Caspi, M. Helm, and L. J. Laslett, "An approach to 3d magnetic field calculation using numerical and differential algebra methods," Lawrence Berkeley Laboratory, LBL-32624, SC-MAG-395, July 1992.

[3] S. Caspi and C. Taylor, "Field harmonics in the $18 \mathrm{~cm}$ wide superbend dipole magnet," Lawrence Berkeley Laboratory, SC-MAG-494, March 1995. 